\title{
Studies of the Petrov module for a family of generalized Liénard integrable systems
}

\begin{abstract}
In this article we use the Lambert function in order to study a family of integrable generalized Liénard equations $X_{f}$ which display a center. We first prove a conjugation lemma inside a continuum of nested periodic orbits. Then we deduce an explicit operator of Gelfand-Leray associated with the Hamiltonian of equation $X_{f}$. Afterwards, we provide a generating family for the associated Petrov module. Finally, by using the Lambert function, we study the monotonicity of the Abelian integral of this generating family's elements.
\end{abstract}

Mathematics Subject Classification (2010). 34C05 and 34A34 and 34C14.

Keywords. Liénard systems and Abelian integrals and Lambert function.

\section{Lucile Mégret}

lucile.megret@upmc.fr

\section{Introduction}

The simplified version of the second part of Hilbert's $16^{\text {th }}$ problem is related to the least upper bound on the number of limit cycles of Liénard system of type

$$
\begin{aligned}
& \dot{x}=y-F(x) \\
& \dot{y}=-g(x),
\end{aligned}
$$

$g(x)$ and $F(x)$ are polynomials.

There are lot of works about the simplified version of Hilbert's $16^{\text {th }}$ problem and related topics see $[18,24]$.

The prolongation of limit cycles in planar vector fields has been widely studied, both by analytical and numerical techniques, see for exemple [19, 20, 4, $5,3,9,11]$. This article deals with integrable systems which display a center surrounded by a graph

$$
\gamma=\partial D
$$

which is a bounded or unbounded heteroclinic or homoclinic connection. We assume furthermore that the integrable system displays a continuum of nested periodic orbits in the domain $D$. The general problem is how to prolong the cycles that arise near the center up to the graph. This problem has been fully investigated recently by Iliev-Gavrilov [14], 2015. 
In this article, we consider a family of integrable generalized Liénard equations of the form

$$
\begin{aligned}
& \dot{x}=y-f(x) \\
& \dot{y}=f^{\prime}(x)
\end{aligned}
$$

where $f=x^{2} / 2+O\left(x^{3}\right)$ is a polynomial function. This equation admits the function

$$
H(x, y)=e^{-y}(f(x)-y-1)
$$

as a first integral.

In particular, the case $f(x)=\frac{1}{2} x^{2}$ has been investigated recently by JeanPierre Françoise and Dongmei Xiao in [12] (2015), where they propose the use of the Lambert function. A simplification of the problem is based on an explicit global change of coordinates which linearizes globally equation (1) (with $f=x^{2} / 2$ ), and relies on the use of the Lambert function. As a corollary, they find an explicit operator of Gelfand-Leray associated with the Darboux first integral $H(x, y)=e^{-y}\left[x^{2} / 2-y-1\right]$. Here, we generalize this result, obtained in [12] with the following theorem:

Theorem 1. Let us consider system (1). Let $f\left(x_{0}\right)$ be a local minimum of $f$ surrounded by a continuum of nested periodic orbits, and let $h_{\max }$ be the value such that the level set $H(x, y)=h_{\max }$ is a bounded (or unbounded) heteroclinic or homoclinic connection which limits this continuum. Let I be an open interval containing $x_{0}$ and such that $f / f^{\prime}$ is analytic on $I$. We define $J$ as the interval such that $(x \in I) \cap(H(x, y) \in]-1, h_{\max }[) \Rightarrow y \in J$. Given any analytic 1-form $\alpha=f(x, y) d x+g(x, y) d y$ with $\omega=d \alpha=F(x, y) d x \wedge d y$, its associated Gelfand-Leray 1-form relatively to $H$ on the rectangle $I \times J$ is given by:

$$
\eta=\frac{F(x, y)}{1+h}\left(\frac{f(x)}{f^{\prime}(x)} \frac{e^{y}-1}{y} d y-\frac{e^{y}-1-y}{y} d x\right) .
$$

The classical results on perturbation theory of integrable systems by a polynomial perturbation (say of fixed degree), reduces the search field of limit cycles in the interior period domain by finding the zeros of the so-called bifurcation function. This bifurcation function, at the leading order of the perturbation, is an integral along the level sets of $H$. These integrals are called Abelian integral. Li and Zhang have provided in [17] a criterion which gives a sufficient condition for the monotonicity of the ratio of two Abelian integrals. This criterion is used in several papers dealing with zeros of Abelian integrals (see $[6,7,8,22]$ for instance). Obviously, exact forms and forms proportional to $d H$ have zero Abelian integrals, so that Abelian integrals depend on the class of forms in the so-called Petrov module. It represents the quotient of the space of all forms by a subspace spanned by exact forms and forms proportional to $d H$, introduced by Petrov in [23] (1987). Therefore the analysis of this module is a key element to track the limit cycles which emerge from a perturbation of an integrable dynamical system. We develop this approach in the last section of the article. 
Outline. The outline of the paper is as follows. In Section 2 we introduce the parameterization of the level set $H=h$ with the Lambert function, and we state some technical lemmas useful for the following. In section 3, we exhibit a change of variables locally analytic which linearizes equation (1), allowing us to prove theorem (1). In section 4, we provide an infinite-dimensional generating family of the Petrov module, and study the monotonicity of the Abelian integral of its elements.

\section{Parameterization with the Lambert function}

For the reader's convenience, we recall here some properties of the Lambert function. These results can be found in [1].

Let us consider the function:

$$
f: u \mapsto z=u e^{u} .
$$

This function is locally invertible in the region of $u=0$. The Lagrange inversion theorem yields as inverse the series

$$
W_{0}(z)=\sum_{n=1}^{+\infty} \frac{(-n)^{n-1}}{n !} z^{n} .
$$

This convergent series is called the principal branch of the Lambert function $[1]$.

Note that $f$ displays a critical point at $u=1$, hence a critical value at $z=e^{-1}$. This implies that the convergence radius upper bound is $e^{-1}$. In fact the Cauchy criterion gives that the radius of convergence is exactly $e^{-1}$. The following lemma is proved in [1] (p. 23).

Lemma 1. The Lambert function displays a square-root branching point at point $z=-e^{-1}$. More precisely, if we change variable $z$ into $p=\sqrt{2(\mathrm{e} z+1)}$, there is a convergent series on the disc $p \in D(0, \sqrt{2})$ such that:

$$
W_{0}(z)=-1+\Phi(p)=-1+p-\frac{1}{3} p^{2}+\frac{11}{72} p^{3}+\ldots
$$

The square-root branching point given by the principal branch of the Lambert function $W_{0}(z)$ generates another analytical branch of the Lambert function

$$
W_{-1}(z)=-1+\phi(-p) .
$$

The relation between the two branches $W_{-1}$ and $W_{0}$ near the branch point was investigated by Karamata [16].

There is an infinity of complex branches of the Lambert function which are solutions of the equation $u e^{u}=z$. Only two, $W_{0}$ and $W_{-1}$, are real. Figure 1 shows the two real branches of the Lambert function. In our case, we only need this two real branches. 


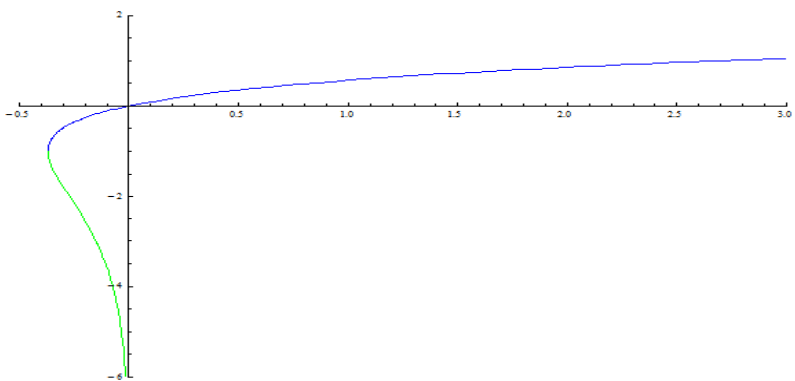

Figure 1. The two real branches of the Lambert function on $]-1 / e,+\infty\left[\right.$. In blue the principal branch $W_{0}$, in green $W_{-1}$.

As stated in the introduction, the integrable dynamical system (1)

$$
\begin{aligned}
& \dot{x}=y-f(x) \\
& \dot{y}=-f^{\prime}(x)
\end{aligned}
$$

admits the first integral $H(x, y)=e^{-y}[f(x)-y-1]$, for any polynomial $f(x)$. We suppose that $f=x^{2} / 2+O\left(x^{3}\right)$. A point is called a Morse point if and only if it is a critical point with non-zero Hessien. We have the follwing lemma:

Lemma 2. The critical points of the Hamiltonian function $H$ are exactly the points $\left(x_{0}, y_{0}\right)$ such that $f^{\prime}\left(x_{0}\right)=0$ and $y_{0}=f\left(x_{0}\right)$. Critical values of $H$ are $h_{0}=-\exp \left(-f\left(x_{0}\right) / \varepsilon\right)$. The critical points of $H$ are Morse points either of center type (for local minima of $f(x)$ ) or of saddle type (for local maxima of $f(x))$.

Proof. Lemma 2. If we choose local coordinates centered at $\left(x_{0}, y_{0}=f\left(x_{0}\right)\right)$ by posing:

$$
\begin{aligned}
& x=x_{0}+X \\
& y=f\left(x_{0}\right)+Y,
\end{aligned}
$$

The Taylor expansion of the Hamiltonian at $\left(x_{0}, y_{0}\right)$ yields:

$$
H_{\varepsilon}(X, Y)=\mathrm{e}^{-f\left(x_{0}\right)}\left[-1+\frac{f^{\prime \prime}\left(x_{0}\right)}{2} X^{2}+2 Y^{2}+\ldots\right]
$$

and this shows the lemma.

Note that closed level sets of the function $H$ cannot intersect the curve $y=f(x)-1$ (which corresponds to $H=0$ ). Since these sets must contain a critical point of $H$ in their interiors, the closed orbits are necessarily all above the slow curve, in the domain union of $\{H=h<0\}$. Furthermore, the Morse lemma shows that all orbits close to a critical point associated with a minimum of $f$ are necessarily closed. The phase portrait of the Hamiltonian of $H$ displays nested periodic orbits around each critical point of center type. The topological type of the level sets of $\{H=h\}$ can change only when $h$ 

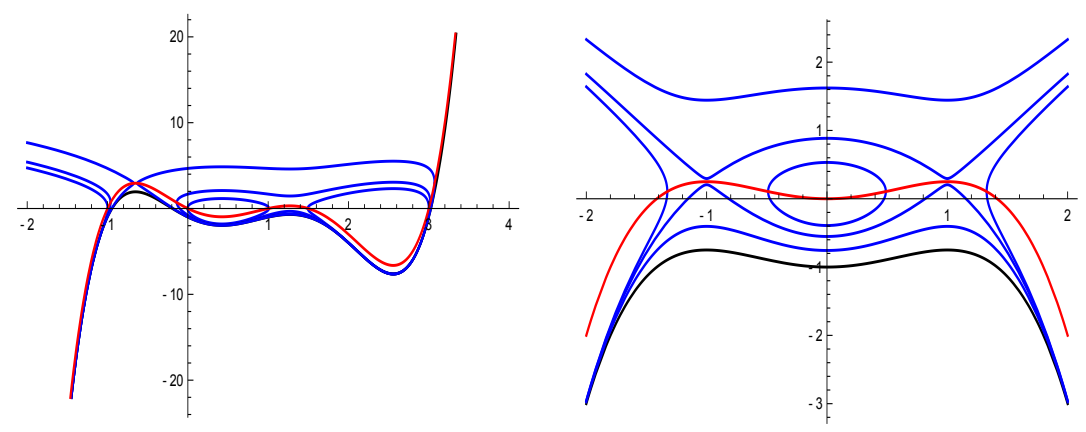

Figure 2. Right: phase portrait of (1) for $f(x)=(x+$ 1) $x(x-1)(x-3 / 2)(x-3)$, left: phase portrait of $(1)$ for $f(x)=$ $x^{2} / 2-x^{4} / 4$. The black curve represents the particular orbit $y=f(x)-1$ obtained for $h=0$.

crosses a critical value. Such critical levels correspond to homoclinic or heteroclinic loops. In some cases, a continuum (nest) of periodic orbits bounded by a heteroclinic loop can be included in another nest of periodic orbits as seen below.

Setting $u=-y-f(x)-1$, we obtain:

$$
u e^{u}=\frac{h}{e} e^{f(x)} .
$$

Therefore, the trajectories can be divided in two parts

$$
\begin{aligned}
& y_{+}(x, h)=f(x)-1-W_{-1}\left(\frac{h}{e} \mathrm{e}^{f(x)}\right) \text { above the curve } y=f(x), \\
& y_{-}(x, h)=f(x)-1-W_{0}\left(\frac{h}{e} \mathrm{e}^{f(x)}\right) \text { below the curve } y=f(x) .
\end{aligned}
$$

Differentiating the expression of $y(x)$, we verify that periodic orbits intersect transversally the critical curve into exactly two points. Hence, we can write that for any trajectory $\gamma$ of system $(1), y=f(x)-1-W\left(\frac{h}{e} e^{f}(x)\right)$ where $W$ is $W_{-1}$ above the curve $y=f(x)$ and $W_{0}$ below the curve $y=f(x)$.

For any nest of periodic orbits corresponding to $H(x, y)=h \in] h_{\min }, h_{\max }[$, we will note $x^{-}(h)$ and $x^{+}(h)$ the points such that $x^{-}(h)<x^{+}(h)$ and

$$
\begin{aligned}
& y_{+}\left(x^{-}(h), h\right)=y_{-}\left(x^{-}(h), h\right)=f\left(x^{-}(h)(h)\right), \\
& y_{+}\left(x^{+}(h), h\right)=y_{+}\left(x^{-}(h), h\right)=f\left(x^{+}(h)(h)\right) .
\end{aligned}
$$

We have $f\left(x^{ \pm}(h)\right)=-\ln (-h)$.

If we consider a nest of periodic orbits surrounding a local minimum, we can see that $x^{-}(h)$ and $x^{+}(h)$ are from either side of the local minimum. It implies that $f^{\prime}\left(x^{-}(h)\right)<0$ and $f^{\prime}\left(x^{+}(h)\right)>0$.

If we consider a nest of periodic orbits surrounding two or more other nests of periodic orbits, we still have $f^{\prime}\left(x^{-}(h)\right)<0$ and $f^{\prime}\left(x^{+}(h)\right)>0$. Indeed, the nest considered has to be bounded in a local maximum or to be unbounded. 
Lemma 3. Suppose that there is a continuum of periodic orbits corresponding to the level sets of $H(x, y)=h$ with $h \in] h_{\min }, h_{\max }[$. Then the functions, $h \rightarrow x^{+}(h)$ defined on $] h_{\min }, h_{\max }[$ are increasing and the function $h \rightarrow$ $x^{-}(h)$ defined on $] h_{\min }, h_{\max }[$ are decreasing.

In addition, an unbounded nest, characterized by $h_{\max }=0$, leads to

$$
\lim _{h \rightarrow 0} x^{ \pm}(h)= \pm \infty
$$

Proof. Lemma 3. To prove the monotonicity of $x^{ \pm}(h)$, we differentiate the expression of $f\left(x^{ \pm}(h)\right)$ with respect to $h$. We get

$$
\frac{d x^{+}}{d h}(h)=\left(x^{+}\right)^{\prime}(h) f^{\prime}\left(x^{+}(h)\right)=-1 / h>0
$$

and

$$
\frac{d x^{-}}{d h}(h)=\left(x^{-}\right)^{\prime}(h) f^{\prime}\left(x^{-}(h)\right)=-1 / h>0 .
$$

We know that $f^{\prime}\left(x^{+}(h)\right)>0$ and $f^{\prime}\left(x^{-}(h)\right)<0$. The conclusion follows.

In order to prove that $\lim _{h \rightarrow 0}^{\rightarrow} x^{ \pm}(h)= \pm \infty$, we just need to note that the level set $H(x, y)=h$ converges to $y=f(x)-1$ when $h$ goes to zero.

With these last results, we can provide a new proof of the following proposition

Proposition 1. Let us consider a nest of periodic orbits surrounding a local minima $\left(x_{0}, f\left(x_{0}\right)\right)$. Let $\left[-1, h_{\max }[\right.$ be the interval such that the level sets $H(x, y)=h$ surrounding $\left(x_{0}, f\left(x_{0}\right)\right)$ are closed for every $h \in\left[-1, h_{\max }[\right.$. Then, the period of the limit cycle converges to $2 \pi$ when the periodic orbits go to the equilibrium point. Let $T(h)$ be the period of the limit cycle corresponding to $H(x, y)=h$. We have:

$$
\lim _{h \rightarrow-1} T(h)=2 \pi .
$$

In addition, if $h_{\max }=0$ (that is, if the nest is unbounded),

$$
\lim _{h \rightarrow 0} T(h)=+\infty .
$$

Proof. Proposition 1. We express $T(h)$ with the help of the Lambert principal branch and $W_{-1}$ :

$$
\begin{aligned}
T(h) & =\int_{0}^{T(h)} d t \\
& =\int_{x^{-}(h)}^{x^{+}(h)} \frac{1}{1+W_{0}\left(\frac{h}{e} e^{x^{2} / 2}\right)}-\frac{1}{1+W_{-1}\left(\frac{h}{e} e^{x^{2} / 2}\right)} d x \\
& =\int_{x^{-}(h)}^{x^{+}(h)} F(x, h) d x .
\end{aligned}
$$


Since $1 \geq 1+W_{0}(X) \geq 0$ for all $X \in\left[-e^{-1}, 0\right]$ and that $1+W_{-1}(X)<0$ for all $X \in\left[-e^{-1}, 0[\right.$, it is clear that

$$
F(x, h)>1 .
$$

In addition, we have proved in lemma 3 that $\left|x^{ \pm}(h)\right|$ goes to infinity when $h \rightarrow 0$. We get $\lim _{h \rightarrow 0} T(h)=+\infty$.

We shall now prove that $\lim _{h \rightarrow-1} T(h)=2 \pi$. To this end, we will use the development of the Lambert function in $-e^{-1}$. We recall that, when $z \rightarrow-e^{-1}$, we have:

$$
\begin{gathered}
W_{-1}(z)=-1-p+O\left(p^{2}\right), \\
W_{0}(z)=-1+p+O\left(p^{2}\right),
\end{gathered}
$$

where $p=\sqrt{2(e z+1)}$. In addition, the flow of system (1) close to a center is equivalent to the flow of

$$
\begin{aligned}
& \dot{x}=y-x^{2} / 2 \\
& \dot{y}=-x .
\end{aligned}
$$

Consequently, we will study the period close to the origin for the solutions of system (11).

We introduce $l(h)=\sqrt{-2 \ln (-h)}$. We get

$$
\begin{aligned}
T(h) & =\int_{0}^{T(h)} d t \\
& =2 \int_{0}^{l(h)} \frac{1}{1+W_{0}\left(\frac{h}{e} e^{x^{2} / 2}\right)}-\frac{1}{1+W_{-1}\left(\frac{h}{e} e^{x^{2} / 2}\right)} d x \\
& =4 \int_{0}^{l(h)} \frac{1}{\sqrt{2\left(h e^{x^{2} / 2}+1\right)}}\left(1+O\left(p^{2}\right)\right) d x \\
& =2 \sqrt{2} \int_{0}^{l(h)} \frac{1}{\sqrt{1+h\left(1+x^{2} / 2+O\left(x^{4}\right)\right)}}\left(1+O\left(p^{2}\right)\right) d x .
\end{aligned}
$$

Let us pose $h=-1+\delta$. Then, $l(h)=\sqrt{2 \delta}(1+O(\sqrt{\delta})$. It follows:

$$
\begin{aligned}
T(h) & =2 \sqrt{2} \int_{0}^{\sqrt{2 \delta}(1+O(\sqrt{\delta}))} \frac{1}{\sqrt{\left.\delta+h \frac{x^{2}}{2}+O\left(x^{4}\right)\right)}}(1+O(\delta)) d x \\
& =2 \sqrt{2} \int_{0}^{\sqrt{2 \delta}(1+O(\sqrt{\delta}))} \frac{1}{\sqrt{\delta+h \frac{x^{2}}{2}}} \frac{(1+O(\delta))}{1+O\left(x^{4} /\left(\delta+h x^{2} / 2\right)\right)} d x \\
& =2 \sqrt{2} \int_{0}^{\sqrt{2 \delta}(1+O(\sqrt{\delta}))} \frac{1}{\sqrt{\delta+h \frac{x^{2}}{2}}} \frac{(1+O(\delta))}{1+O\left(x^{3}\right)} d x \\
& =2 \sqrt{2} \int_{0}^{\sqrt{2 \delta}(1+O(\sqrt{\delta}))} \frac{1}{\sqrt{\delta+h \frac{x^{2}}{2}}}\left(1+O\left(x^{3}\right)\right) .
\end{aligned}
$$


Because $x^{2} / 2 \leq \delta(1+O(\sqrt{\delta}))$, for $\delta$ small enough, there exist two constants $C_{1}, C_{2}$ such that:

$$
\begin{array}{r}
2 \sqrt{2} \int_{0}^{\sqrt{2 \delta}(1+O(\sqrt{\delta}))} \frac{1}{\sqrt{\delta+h \frac{x^{2}}{2}}}\left(1+C_{1} \delta^{3}\right) \leq T(h) \\
\leq 2 \sqrt{2} \int_{0}^{\sqrt{-2 \ln (-h)}} \frac{1}{\sqrt{\delta+h \frac{x^{2}}{2}}}\left(1+C_{2} \delta^{3}\right) .
\end{array}
$$

This integral can be directly solved using classical primitive functions:

$$
\begin{aligned}
& 2 \sqrt{2} \int_{0}^{\sqrt{2 \delta}(1+O(\sqrt{\delta}))} \frac{1}{\sqrt{\delta+h \frac{x^{2}}{2}}}\left(1+C \delta^{3}\right) \\
= & 2 \sqrt{\frac{2}{\delta}}\left(1+C \delta^{3}\right) \int_{0}^{\sqrt{2 \delta}(1+O(\sqrt{\delta}))} \frac{1}{\sqrt{1-\left(\sqrt{\frac{|h|}{2 \delta}} x\right)^{2}}} \\
= & \sqrt{\frac{2}{|h|}}\left(1+C \delta^{3}\right) \arcsin (\sqrt{|h|}(1+O(\sqrt{\delta}))) .
\end{aligned}
$$

We conclude that

$$
\lim _{h \rightarrow-1} T(h)=2 \pi
$$

Despite these results on the asymptotic periods, we still have no result of monotonicity. Actually, we can prove that, in some case, the period of the limit cycles inside a nest of periodic orbits cannot evolve in a monotonic way with respect to $h$. Indeed, the following lemma can be proved:

Lemma 4. Suppose that, for $h^{*}$, one of the level set $H(x, y)=h^{*}$ is a homoclinic or a heteroclinic loop. Then, $\lim _{h \rightarrow h^{*}} T(h)=+\infty$.

It is well known that the period of a homoclinic loop is infinite. We can obtain an additional proof by verifying that the singularity of

$$
\int_{x^{-}(h)}^{x^{+}(h)} \frac{1}{1+W_{0}\left(\frac{h}{e} e^{x^{2} / 2}\right)}-\frac{1}{1+W_{-1}\left(\frac{h}{e} e^{x^{2} / 2}\right)} d x
$$

in $x^{+}(h)$ and in $x^{-}(h)$ are not integrable if $\left(x^{ \pm}(h), f\left(x^{ \pm}(h)\right)\right)$ is an equilibrium point of saddle type.

A consequence of lemma 4 , is that the period can not be strictly increasing on a nest surrounding a homoclinic or heteroclinic connection.

\section{A conjugation lemma}

In this section, we will prove theorem 1.

We will first prove a local version of this theorem. We will provide a local change of coordinates which linearize equation (1). Then we will deduce an explicit operator of Gelfand-Leray associated with the Hamiltonian $e^{-y}(f(x)-y-1)$. 
We suppose that for all $h \in\left[-1, h_{\max }[\right.$, the level sets $H(x, y)=h$ are closed and surround the origin. Let us define

$$
I=] x_{0}, x_{1}[\ni 0,
$$

as an open interval containing zero and such that $f / f^{\prime}$ is analytic on $I$. It means that either $x_{0}=x^{-}\left(h_{\max }\right)$, or $x_{1}=x^{+}\left(h_{\max }\right)$. Hence function $f$ is strictly decreasing on $\left.]-x_{0}, 0\right]$ and strictly increasing on $\left[0, x_{1}[\right.$. Let us define the reciprocal of $f$ on both intervals. We write:

$$
u \rightarrow f_{s g(u)}^{-1}(u),
$$

where $s g(u)$ is the sign of $u$. We define $f_{-}^{-1}$ as the reciprocal of $f$ on $\left.]-x_{0}, 0\right]$ and $f_{+}^{-1}$ as the reciprocal of $f$ on $\left.] 0, x_{1}\right]$.

Let

$$
J=]-1-W_{0}\left(h_{\max } e^{-1}\right),-1-W_{-1}\left(h_{\max } e^{-1}\right)[=] y_{\min }, y_{\max }[
$$

be the interval such that for all $x \in I$ and for all $h \in]-1, h_{\max }[$, $H(x, y)=h \Rightarrow y \in J$.

We recall that in a neighborhood of $z=-e^{-1}$, by posing $p=\sqrt{2(\mathrm{e} z+1)}$, we have

$$
W_{0}(z)=-1+\Phi(p)=-1+p+O\left(p^{2}\right)
$$

and

$$
W_{0}(z)=-1+\Phi(p)=-1-p+O\left(p^{2}\right) .
$$

We can now state the following theorem

Theorem 2. The change of variables

$$
\begin{aligned}
F: \quad \Omega=I \times] \Phi^{-1}\left(-y_{\max }\right), \Phi^{-1}\left(-y_{\min }\right)[ & \rightarrow I \times J \\
(X, Y) & \rightarrow(x, y)
\end{aligned}
$$

where

$$
\left\{\begin{aligned}
x & =f_{s g(X)}^{-1}\left[e^{s g(Y) \Phi(-Y)} f(X)\right] \\
y & =s g(Y) \Phi(-Y)
\end{aligned}\right.
$$

is analytical and satisfies:

$$
H(x, y)=e^{-y}[f(x)-y-1]=f(X)+\frac{Y^{2}}{2}-1 .
$$

The inverse of this application is

$$
\begin{aligned}
& F^{-1}:(x, y) \rightarrow(X, Y) \\
& \left\{\begin{array}{l}
X=f_{s g(x)}^{-1}\left[e^{-y} f(x)\right] \\
Y=T(y)
\end{array}\right.
\end{aligned}
$$

where

$$
T(y)=\left\{\begin{array}{cl}
\sqrt{2\left[1-(1+y) e^{-y}\right]} & \text { if } y>0 \\
0 & \text { if } y=0 \\
-\sqrt{2\left[1-(1+y) e^{-y}\right]} & \text { if } y>0
\end{array} .\right.
$$


Proof. Theorem 2 Now, let us prove that $F^{-1}$ is well defined.

We define:

$$
z:=\frac{1}{e}\left(\frac{Y^{2}}{2}-1\right)=p^{-1}(Y) .
$$

By definition of $\Phi$, for $-\sqrt{2}<Y<\sqrt{2}$, we have

$$
-(1+y)=W_{0}\left(p^{-1}(-Y)\right)=-1+\Phi(|Y|) \text { for } y<0
$$

and

$$
-(1+y)=W_{-1}\left(p^{-1}(-Y)\right)=-1+\Phi(-|Y|) \text { for } y>0 .
$$

Hence, we have $] \Phi^{-1}\left(-y_{\max }\right), \Phi^{-1}\left(-y_{\min }\right)[\subset]-\sqrt{2}, \sqrt{2}[$. In addition, it follows that

$$
p^{-1}(Y)=-(1+y) e^{-(1+y)}
$$

and that, $y$ and $Y$ have the same sign.

We can now verify that $F^{-1}$ is well defined. We have

$$
0 \leq e^{s g(Y) \Phi(-Y)} f(X) \leq f(X),
$$

therefore

$$
f_{s g(X)}^{-1}\left[e^{s g(Y) \Phi(-Y)} f(X)\right]
$$

is well defined.

It is clear that $e^{-y} f(x)=f(X)$ and $-e^{-y}(y+1)=Y^{2} / 2-1$. Hence, $H(x, y)=$ $f(X)+Y^{2} / 2-1$.

It follows that $F^{-1}$ is the reciprocal of $F$ :

Let us consider the vector field defined on $\Omega$

$$
D=2 \frac{f(X)}{f^{\prime}(X)} \frac{\partial}{\partial X}+Y \frac{\partial}{\partial Y} .
$$

It satisfies the relation

$$
\text { D. } H=2 H+2 .
$$

By computing $D$ in the original coordinates, we obtain the following results as the corollary of theorem 2 .

Corollary 1. The vector field defined on $I \times \mathbb{R}$

$$
D=2 \frac{f(x)}{f^{\prime}(x)} \frac{e^{y}-1}{y} \frac{\partial}{\partial x}+2 \frac{e^{y}-y-1}{y} \frac{\partial}{\partial y} .
$$

satisfies

$$
\text { D. } H=2 H+2,
$$

where $(x, y) \rightarrow H(x, y)=e^{-y}(f(x)-y-1)$.

We deduce an explicit expression of the Gelfand-Leray 1-form associated with $H$. That is, we find a 1 -form $\eta$ such that for any

$$
d \alpha=G(x, y) d x \wedge d y
$$

where $G$ is analytic, we have $d \alpha=d H \wedge \eta$. 
Lemma 5. Let us consider the integrable generalized Liénard system (1)

$$
\begin{aligned}
\dot{x} & =y-f(x) \\
\dot{y} & =-f^{\prime}(x) \\
f(x) & =x^{2} / 2+O\left(x^{3}\right),
\end{aligned}
$$

where $f(x)$ is a polynomial function. Let $I$ be the open interval containing the origin such that $f(x) / f^{\prime}(x)$ is analytic on $I$. Let

$$
\alpha=s(x, y) d x+r(x, y) d y
$$

be an analytic 1 -form defined on $I \times \mathbb{R}$. We can find an analytic function $F$ such that

$$
\omega=d \alpha=F(x, y) d x \wedge d y .
$$

Its associated Gelfand-Leray 1-form relatively to $H$ is:

$$
\eta=\frac{F(x, y)}{1+h}\left(\frac{f(x)}{f^{\prime}(x)} \frac{e^{y}-1}{y} d y-\frac{e^{y}-1-y}{y} d x\right) .
$$

Proof. Llemma 5 The proof of the lemma 5 is obtained by showing that

$$
\omega=d H \wedge \eta \text {. }
$$

We immediately get the result.

Lemma 5 can be generalized at every nest of periodic orbits. Indeed, let us suppose that $f$ admits another local minimum at $\left(x_{1}, f\left(x_{1}\right)\right)$. Then, this point is surrounded by a nest of periodic orbits corresponding to $H(x, y) \in$ ]$-1, h_{\max , x_{1}}\left[\right.$. We can define a reciprocal of $f u \rightarrow f_{s g\left(x_{1}-u\right), x_{1}}^{-1}(u)$ in the same way as we have defined $f^{-1}$ in equation (17).

Let $I_{x_{1}}$ be the interval such that $f(x)-f\left(x_{1}\right) / f^{\prime}(x)$ is analytical on $I_{x_{1}}$.

Let $J_{x_{1}}$ be the interval such that $\left.\left(x \in I_{x_{1}}\right) \cap(H(x, y)) \in\right]-1, h_{\max , x_{1}}[\Rightarrow y \in$ $J_{x_{1}}$.

The vector field defined on $I_{x_{1}} \times J_{x_{1}}$ by

$$
D_{x_{1}}=2 \frac{f(x)-f\left(x_{1}\right)}{f^{\prime}(x)} \frac{e^{y}-1}{y} \frac{\partial}{\partial x}+2 \frac{e^{y}-y-1}{y} \frac{\partial}{\partial y} .
$$

satisfies

$$
D_{x_{1}} \cdot H=2 H+2\left(1-f\left(x_{1}\right)\right) .
$$

The GelfandLeray 1-form associated with $H$ is still given by:

$$
\eta=\frac{F(x, y)}{1+h}\left(\frac{f(x)}{f^{\prime}(x)} \frac{e^{y}-1}{y} d y-\frac{e^{y}-1-y}{y} d x\right) .
$$

Indeed, we still have

$$
\omega=d H \wedge \eta
$$

on $I_{x_{1}} \times J_{x_{1}}$. In addition, for all $\left.h \in\right]-1, h_{\max , x_{1}}\left[\right.$, the function $f(x) / f^{\prime}(x)$ is well defined and analytical on the level set $H(x, y)=h$.

Let us now suppose that the nest of periodic orbits surrounding $\left(x_{1}, f\left(x_{1}\right)\right)$ ends in a homoclinic loop in the same point as the nest surrounding the origin. In this case, we have $h_{\max , x_{1}}=h_{\max }$ as illustrated in figure 3 . Let us define interval $I_{3}$ such that for all $x \in I_{3}$ the function $\left(f(x)-f\left(x_{1}\right) f(0)\right) / f^{\prime}(x)$ is 
analytical. For all $h$ with $h_{\max }<h<h_{\max }^{*}$ and $x \in I_{3}$, there is a unique closed level set $H(x, y)=h$. This level set surrounds the two nests of periodic orbits which end in $H(x, y)=h_{\max }$. Let us define $J_{3}$ as the interval such that $\left.\left(x \in I_{3}\right) \cap(H(x, y)) \in\right] h_{\max }, h_{\max }^{*}\left[\Rightarrow y \in J_{3}\right.$.

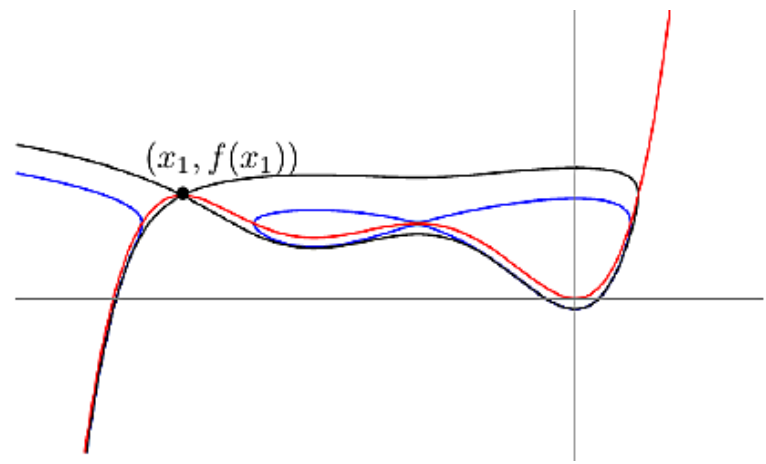

Figure 3. In red the curve $y=f(x)$, in blue the level set $H(x, y)=h_{\max }=h_{\max , x_{1}}$, in black the level set $H(x, y)=$ $h_{\max }^{*}$.

We can define piecewise a reciprocal of $f$ on $I_{3}$ and

$$
D_{0, x_{1}}=2 \frac{f(x)-f\left(x_{1}\right) f(0)}{f^{\prime}(x)} \frac{e^{y}-1}{y} \frac{\partial}{\partial x}+2 \frac{e^{y}-y-1}{y} \frac{\partial}{\partial y} .
$$

satisfies

$$
D_{0, x_{1}} \cdot H=2 H+2\left[1-f\left(x_{1}\right) f(0)\right] .
$$

Again, the GelfandLeray 1-form associated with $H$ is given by:

$$
\eta=\frac{F(x, y)}{1+h}\left(\frac{f(x)}{f^{\prime}(x)} \frac{e^{y}-1}{y} d y-\frac{e^{y}-1-y}{y} d x\right) .
$$

We can proceed this way in order to prove that $\eta$ is the GelfandLeray 1-form associated with $H$ on any nest of periodic orbits. This completes the proof of theorem (1).

\section{Petrov module associated with the Hamiltonian function $H(x, y)=e^{-y}(f(x)-y-1)$}

L. Gavrilov proved in [13] that the Petrov module of a generic Hamiltonian is a finitely generated free $\mathrm{C}[\mathrm{t}]$-module. In our case the degree of the Hamiltonian $H$ is not finite, we cannot conclude that a finite generating family of the Petrov module exists. Nevertheless, J.-P. Françoise and D. Xiao have proved in [12] that for $f(x)=x^{2} / 2$, the family of 1 -form $\left(\omega_{j}=y^{j} d x\right)_{1 \leq j}$ defines a generating family of the Petrov module,

$$
\Omega_{r e l}=\Omega^{1} / g d H+d R,
$$


associated with $H(x, y)=e^{-y}\left(x^{2} / 2-y-1\right)$. We can generalize this result to any polynomial $f(x)=x^{2} / 2+O(x)^{3}$.

Theorem 3. Let us consider system (1) where $f=x^{2} / 2+a_{3} x^{3}+\ldots+a_{n} x^{n}$ is a polynomial function of degree $n \geq 3$.

The Petrov module,

$$
\Omega_{r e l}=\Omega^{1} / g d H+d R
$$

is infinitely generated by the family of 1-forms

$$
\Omega_{n}=\left(\omega_{p, j}=x^{p} y^{j} d x\right)_{0 \leq p \leq n-2, j \geq 1} .
$$

Proof. Theorem 3. We refer to the relation $\omega \equiv \omega^{\prime}$ if we can find two analytical functions $g$ and $R$ such that $\omega-\omega^{\prime}=g d H+d R$.

- First, let us show that every 1 -form $x^{i} y^{j} d x$ can be written as a convergent series of terms belonging to $\Omega_{n}$. We will proceed by induction on the degree of $x$. Let us begin with $p=n-1$ :

$$
\begin{aligned}
x^{n-1} y^{j} d x & =\frac{1}{n a_{n}} y^{j} d\left(a_{n} x^{n}\right) \\
& =\frac{1}{n a_{n}} y^{j} d\left(H e^{y}-y+1-\left(x^{2} / 2+a_{3} x^{3}+\ldots+a_{n-1} x^{n-1}\right)\right) \\
& \equiv-\frac{1}{n a_{n}} y^{j}\left(x+3 a_{3} x^{2}+\ldots+(n-1) a_{n-1} x^{n-2}\right) d x,
\end{aligned}
$$

Therefore the property is verified for $x^{n-1} y^{j} d x$. We can now show that the property is inductive. Suppose that for some $N \geq n-1 \geq 2, \forall k \leq N, x^{k} y^{j} d x$ can be written as a convergent series of the terms of $\omega_{p, j}$. Then we obtain

$$
\begin{aligned}
x^{N+1} y^{j} d x= & \frac{1}{n a_{n}} x^{N-n+2} y^{j} d\left(x^{n}\right) \\
= & \frac{1}{n a_{n}} x^{N-n+2} y^{j} d\left(H e^{y}+y+1-\frac{x^{2}}{3}-\ldots-a_{n-1} x^{n-1}\right) \\
= & \frac{1}{n a_{n}} x^{N-n+2} y^{j} d\left(H e^{y}+y+1\right) \\
& -\frac{y^{j}}{n a_{n}}\left[x^{N-n+3}+3 a_{3} x^{N-n+4}+\ldots+(n-1) a_{n-1} x^{N}\right] d x .
\end{aligned}
$$

The second term of the sum verifies the property by induction.

Let us focus on the first term $x^{N-n+2} y^{j} d\left(H e^{y}+y+1-\frac{x^{2}}{3}-\ldots-a_{n-1} x^{n-1}\right)$. Since $H e^{y}+y+1$ is a convergent series of $y$ and $H$, it is clear that there exists a series $\psi(y, H)$, convergent in $y$ such that

$$
y^{j} d\left(H e^{y}+h+1\right)=d \psi(y, H) .
$$

It follows that

$$
\begin{aligned}
\frac{1}{n a_{n}} x^{N-n+2} y^{j} d\left(H e^{y}+y+1\right)= & \frac{1}{n a_{n}} x^{N-n+2} d(\psi(y, H)) \\
= & d\left(\frac{1}{n a_{n}} x^{N-n+2} \psi(y, H)\right) \\
& -\frac{N-n+2}{n a_{n}} \psi(y, H) x^{N-n+1} d x \\
\equiv & -\frac{N-n+2}{n a_{n}} \psi(y, H) x^{N-n+1} d x .
\end{aligned}
$$

We can apply the induction principle.

- To conclude, let us show that every 1 -forms $x^{i} y^{j} d y$ can be written as a convergent series of terms belonging to $\Omega_{n}$. We remark that $\forall j, y^{j} d y \equiv 0$, and $\forall i \geq 1$

$$
\begin{aligned}
x^{i} y^{j} d y & =\frac{1}{j+1} d\left(x^{i} y^{j+1}\right)-\frac{1}{j+1} y^{j+1} x^{i-1} d x \\
& \equiv-\frac{1}{j+1} y^{j+1} x^{i-1} d x
\end{aligned}
$$


This completes the proof.

This generating family is helpful for investigating the ability of a perturbed system to produce limit cycles. The question is to try to find a bound on the number of zeros of the combinations of the integrals corresponding to the generating family. This is related to important studies made on Tchebycheff property of families of integrals ([15] and [10]).

We will now study the monotonicity of the Abelian integral for the elements of $\Omega_{n}$. The cubic case is of particular interest. Indeed, when perturbed, this system presents a Bogdanov-Takens-SNIC bifurcation, introduced in [21]. Let us consider the system:

$$
\begin{aligned}
& \dot{x}=y-\frac{x^{2}}{2}-\alpha \frac{x^{3}}{3} \\
& \dot{y}=-x-\alpha x^{2} .
\end{aligned}
$$

The Hamiltonian of this equation is given by $H(x, y)=e^{-y}\left(x^{2} / 2+\alpha x^{3} / 3-\right.$ $y-1)$ and the family of 1 -form $\left(y^{j} d x, x y^{j} d x\right)_{j \geq 1}$ generates the associated Petrov module.

We define the functions of $h$ :

$$
h \rightarrow I_{j, n}(h)=\int_{H=h} x^{j} y^{n} d x,
$$

with $j \in\{0,1\}$ and $n \in \mathbb{N}^{*}$. Then, we have the following proposition

Proposition 2. Let us consider system (24). For all $n \geq 1$, the function $h \rightarrow$ $I_{0, n}(h)$ is positive and increasing, the function $h \rightarrow I_{1, n}(h)$ is negative and decreasing if $\alpha>0$, is positive and increasing if $\alpha<0$.

Proof. Proposition 2 We recall that

$$
\begin{aligned}
I_{0, n}(h) & =\int_{\substack{H=h \\
x^{+}}} y(x, h)^{n} d x \\
& =\int_{x^{-}}\left[f(x)-1-W\left(\frac{h}{e} e^{f(x)}\right)\right]^{n},
\end{aligned}
$$


where $\left[f(x)-1-W\left(\frac{h}{e} e^{f(x)}\right)\right]^{n}$ means $\left[f(x)-1-W_{-1}\left(\frac{h}{e} e^{f(x)}\right)\right]^{n}-[f(x)-$ $\left.1-W_{0}\left(\frac{h}{e} e^{f(x)}\right)\right]^{n}$. By differentiating this expression with respect to $h$, we get

$$
\begin{aligned}
I_{0, n}^{\prime}(h) & =\int_{x^{-}}^{x^{+}} \frac{\partial}{\partial h}\left(\left[f(x)-1-W\left(\frac{h}{e} e^{f(x)}\right)\right]^{n}\right) d x \\
& =-\frac{n}{h} \int_{x^{-}}^{x^{+}} \frac{W_{-1}\left(\frac{h}{e} e^{f(x)}\right)}{1+W_{-1}\left(\frac{h}{e} e^{f(x)}\right)}\left[f(x)-1-W_{-1}\left(\frac{h}{e} e^{f(x)}\right)\right]^{n-1} \\
& =-\frac{W_{0}\left(\frac{h}{e} e^{f(x)}\right)}{1+W_{0}\left(\frac{h}{e} e^{f(x)}\right)}\left[f(x)-1-W_{0}\left(\frac{h}{e} e^{f(x)}\right)\right]^{n-1} d x \\
& \int_{x^{-}}^{x^{+}} \frac{W_{-1}\left(\frac{h}{e} e^{f(x)}\right)}{1+W_{-1}\left(\frac{h}{e} e^{f(x)}\right)}\left[y^{+}(x)\right]^{n-1} \\
= & \frac{n}{h} \int_{x^{-}}^{x^{+}}\left|\frac{W_{-1}\left(\frac{h}{e} e^{f(x)}\right)}{1+W_{-1}\left(\frac{h}{e} e^{f(x)}\right)}\right|\left[y^{-}(x)\right]^{n-1} d x \\
&
\end{aligned}
$$

If $n$ is odd, it is clear that $I_{0, n}(h)$ is strictly increasing because every terms are positive.

If $n$ is even, the function $X \rightarrow X^{n-1}$ is strictly increasing on $\mathbb{R}$. Let $n=$ $2(p+1)$ be an even integer. We have, $f(x) \geq 0$. We can find a lower bound for $I_{0, n}^{\prime}(h)$

$$
\begin{aligned}
& \int_{x^{-}}^{x^{+}}\left|\frac{W_{-1}\left(\frac{h}{e} e^{f(x)}\right)}{1+W_{-1}\left(\frac{h}{e} e^{f(x)}\right)}\right|\left[y^{+}(x)\right]^{n-1}+\left|\frac{W_{0}\left(\frac{h}{e} e^{f(x)}\right)}{1+W_{0}\left(\frac{h}{e} e^{f(x)}\right)}\right|\left[y^{-}(x)\right]^{n-1} d x \\
\geq & \int_{x^{-}}^{x^{+}}\left|\frac{W_{-1}\left(\frac{h}{e} e^{f(x)}\right)}{1+W_{-1}\left(\frac{h}{e} e^{f(x)}\right)}\right|\left[-1-W_{-1}\left(\frac{h}{e} e^{f(x)}\right)\right]^{n-1} \\
& \quad+\left|\frac{W_{0}\left(\frac{h}{e} e^{f(x)}\right)}{1+W_{0}\left(\frac{h}{e} e^{f(x)}\right)}\right|\left[-1-W_{0}\left(\frac{h}{e} e^{f(x)}\right)\right]^{n-1} d x \\
= & \int_{x^{-}}^{x^{+}}\left|W_{-1}\left(\frac{h}{e} e^{f(x)}\right)\right|\left|1+W_{-1}\left(\frac{h}{e} e^{f(x)}\right)\right|^{n-2} \\
& \quad+(-1)^{n-1}\left|W_{0}\left(\frac{h}{e} e^{f(x)}\right)\right|\left|1+W_{0}\left(\frac{h}{e} e^{f(x)}\right)\right|^{n-2} d x .
\end{aligned}
$$

We need the following lemma (proved below):

Lemma 6. For all $X \in]-1 / e, 0\left[\right.$ we have $\left|1+W_{0}(X)\right|<\left|1+W_{-1}(X)\right|$.

In addition, it is clear that $\left|W_{-1}(x)\right| \geq\left|W_{0}(x)\right|$. It follows that $I_{0, n}$ is strictly increasing for all $n$. Furthermore, it is easy to check that $I_{1, n}(h)=-\alpha I_{2, n}(h)$. Hence, we obtain

$$
\begin{aligned}
I_{1, n}^{\prime}(h)= & \frac{n \alpha}{h} \int_{x^{-}}^{x^{+}} x^{2} \frac{w_{-1}\left(\frac{h}{e} e^{f(x)}\right)}{1+w_{-1}\left(\frac{h}{e} e^{f(x)}\right)}\left[f(x)-1-W_{-1}\left(\frac{h}{e} e^{f(x)}\right)\right]^{n-1} \\
& -x^{2} \frac{w_{0}\left(\frac{h}{e} e^{f(x)}\right)}{1+w_{0}\left(\frac{h}{e} e^{f(x)}\right)}\left[f(x)-1-W_{0}\left(\frac{h}{e} e^{f(x)}\right)\right]^{n-1} d x .
\end{aligned}
$$

It follows that, $I_{1, n}$ is strictly decreasing if $\alpha>0$, increasing if $\alpha<0$. We remark that $\lim _{h \rightarrow-1} I_{1, n}(h)=\lim _{h \rightarrow-1} I_{0, n}(h)=0$. The conclusion follows. 
Proof. Lemma 6. We just need to study the differences

$$
\left(1+W_{-1}(X)\right)^{2}-\left(1+W_{0}(X)\right)^{2} .
$$

For all $X \in[-1 / e, 0[$ we have

$$
\begin{aligned}
\frac{d(1+W(X))^{2}}{d X} & =2(1+W(X)) \frac{d W(X)}{d X} \\
& =\frac{2}{X} W(X) .
\end{aligned}
$$

Therefore

$$
\frac{d}{d X}\left[\left(1+W_{-1}(X)\right)^{2}-\left(1+W_{0}(X)\right)^{2}\right]=\frac{2}{X}\left(W_{-1}(X)-W_{0}(X)\right)>0 .
$$

It follows that the difference is increasing. Furthermore, we have

$$
\left[1+W_{-1}\left(-e^{-1}\right)\right]^{2}-\left[1+W_{0}\left(-e^{-1}\right)\right]^{2}=0 .
$$

This last equality completes the proof of the lemma.

The proposition 2 can be generalized to some polynomials $f$ under few assumption.

Let us consider system (1) with $f=x^{2} / 2+\hat{f}(x)$ where $\hat{f}(x)=O\left(x^{3}\right)$ is a polynomial function. For all $j \in \mathbb{N}$ and $n \in \mathbb{N}^{*}$ we define the function of $h$ :

$$
h \rightarrow I_{j, n}(h)=\int_{H=h} x^{j} y^{n} d x .
$$

We consider the nest of periodic orbits which contain the origin in their interiors. It correspond to the level sets $H(x, y)=h$ with $h \in\left[-1, h_{\max }[\right.$. Let us pose $x_{\text {end }} \neq 0$ the point such that the loop bounding this nest ends in $\left(x_{\text {end }}, f\left(x_{\text {end }}\right)\right)$ (thus, either $x_{\text {end }}=x^{-}\left(h_{\max }\right)$ or $\left.x_{\text {end }}=x^{+}\left(h_{\max }\right)\right)$. It means that $f\left(x_{\text {end }}\right)$ is the smallest local maximum close to the origin. Suppose that $\hat{f}^{\prime}$ keeps a constant sign on the interval $\left[x^{-}\left(h_{\max }\right), x^{+}\left(h_{\max }\right)\right]$.

We state the following proposition:

\section{Proposition 3.}

1) For all $k \in \mathbb{N}$, the functions $h \rightarrow I_{2 k, n}(h)$ are strictly increasing from $I_{2 k, n}(-1)=0$ to $I_{2 k, n}\left(h_{\max }\right)>0$.

2) For the functions $I_{2 k+1, n}(h)$ there are three possibilities:

- if the system is symmetrical, then $\forall k \in \mathbb{N}, I_{2 k+1, n}(h)=0$;

- if $x_{\text {end }}<0$, then, $\forall k \in \mathbb{N}$, the function $h \rightarrow I_{2 k+1, n}(h)$ is strictly decreasing from $I_{2 k+1, n}(-1)=0$ to $I_{2 k+1, n}\left(h_{\max }\right)<0$;

- if $x_{\text {end }}>0$, then, $\forall k \in \mathbb{N}$, the function $h \rightarrow I_{2 k+1, n}(h)$ is strictly increasing from $I_{2 k+1, n}(-1)=0$ to $I_{2 k+1, n}\left(h_{\max }\right)>0$.

Proof. Proposition 3. For all $j \in \mathbb{N}$, we have

$$
f^{\prime}(x) y^{j} d x=y^{j} d(f(x))=y^{j} d\left(h e^{y}+y+1\right) \equiv 0 .
$$

By setting $f(x)=x^{2} / 2+\hat{f}(x)$, we obtain

$$
x y^{j} d x \equiv-\hat{f}^{\prime}(x) y^{j} d x .
$$

Note that $x=-\hat{f}^{\prime}(x)$ for both $x_{\text {end }}$ and $x=0$. By hypothesis the sign of $\hat{f}$ remains constant. The rest of the proof is exactly the same as in the case $f(x)=x^{2} / 2+\alpha x^{3} / 3$. 
Proposition 4. Let us consider the particular case where system (1) is symmetrical and the nest of periodic orbits is unbounded (for example take $f(x)=$ $\left.x^{2} / 2\right)$. Then, for all $k \in \mathbb{N}$ and $n \in \mathbb{N}^{*}, \lim _{h \rightarrow 0} I_{2 k, n}(h)=+\infty$.

Proof. Proposition 4. For all $k \in \mathbb{N}$ and $n \in \mathbb{N}^{*}$ we have

$$
\begin{aligned}
I_{2 k, n}(h)=2 \int_{0}^{x_{+}(h)} x^{2 k} & {\left[\left(f(x)-1-W_{-1}\left(\frac{h}{e} e^{f(x)}\right)\right)^{n}\right.} \\
& \left.-\left(f(x)-1-W_{0}\left(\frac{h}{e} e^{f(x)}\right)\right)^{n}\right] d x
\end{aligned}
$$

We take into account that $h \rightarrow 0$, we obtain:

$$
\begin{aligned}
& I_{2 k, n}(h)=2 \int_{0}^{x^{+}(h)} x^{2 k} {\left[\left(f(x)-1-W_{-1}\left(\frac{h}{e} e^{x^{2} / 2}\right)\right)^{n}\right.} \\
&\left.-(f(x)-1-O(h))^{n}\right] d x \\
&=2 \int_{0}^{x^{+}(h)} x^{2 k} \sum_{k=0}^{n-1}\left(\begin{array}{c}
n \\
k
\end{array}\right)(f(x)-1)^{k}\left[\left(-W_{-1}\left(\frac{h}{e} e^{f(x)}\right)\right)^{n-k}\right. \\
&\left.-O(h)^{n-k}\right] .
\end{aligned}
$$

Because $x_{+}(h)=f_{+}^{-1}(-\ln (-h)) \underset{h \rightarrow 0}{\rightarrow}+\infty$ and $-W_{-1}\left(\frac{h}{e} e^{f(x)}\right) \underset{h \rightarrow 0}{\rightarrow}+\infty$, the limit of $I_{2 k, n}(h)$ when $h$ goes to zero is $+\infty$.

\section{Conclusion and Perspectives}

In this article we focus on a class of system defining in (1). It is well known that the zeros of Abelian integrals are related to limit cycles emerging via a polynomial perturbation of a polynomial Hamiltonian vector field. Therefore, the ability to differentiate such an integral with respect to $h$ is needed to explore the capacity of system (1) to produce limit cycles under a polynomial perturbation. That is the purpose of the Gelfand-Leray operator, proposed in Theorem 1, which allows us to differentiate the Abelian integral of any 1-form with respect to $h$. Since the Abelian integrals depend on the class of forms in the Petrov module, it is very interesting to know as much as possible about the Abelian integrals of the module's generating family. In their article [12] J.-P. Franoise and D. Xiao provided a Picard-Fuchs equation, associated with the function $H(x, y)=e^{-y}\left(x^{2} / 2-y-1\right)$ based on the Gelfand-Leray operator. When $\omega$ belongs to the generating family of the Petrov module, the PicardFuchs equation gives the differential with respect to $h$ of the Abelian integral,

$$
I(h, \omega)=\int_{H(x, y)=h} \omega,
$$

as a sum of Abelian integrals of the elements of the generating family. Finding a Picard-Fuchs equation associated with the function $H(x, y)=e^{-y}(f(x)-$ 
$y-1$ ) for any polynomial $f(x)=x^{2} / 2+O\left(x^{3}\right)$ can be the object of a future work.

Proposition 3 provides a property of the Petrov module which can be applied to study the monotonicity of the Abelin integral of a perturbation if the integrable system (without the perturbation) verifies some assumption.

It is worth noting that the results obtained in this article can be adapted to the slow-fast case. Which is partially done in [2]. In this article the authors have studied canard solutions of the slow-fast system $\varepsilon \dot{x}=y-x^{2} / 2-\alpha x^{3} / 3$, $\dot{y}=-x-\alpha x^{2}$.

\section{References}

[1] R. M. Corless, G. H. Gonnet, D. E. G. Hare, D. J. Jeffrey, and D. E. Knuth. On the lambert w function. Adv. Comput. Math., 5(1):329-359, 1996.

[2] M. Desroches, J.-P. Franoise, and L. Mégret. Canard-induced loss of stability across a homoclinic bifurcation. ARIMA Rev. Afr. Rech. Inform. Math. Appl., 20:47-62, 2015.

[3] E. J. Doedel, A. R. Champneys, F. Dercole, T. F. Fairgrieve, Y. A. Kuznetsov, B. Oldeman, R. C. Paffenroth, B. Sandstede, X. J. Wang, and C. H. Zhang. Auto-07p: Continuation and bifurcation software for ordinary differential equations. available at the URL http://indy.cs.concordia.ca, 2007.

[4] E. J. Doedel, H. B. Keller, and J.-P. Kernévez. Numerical analysis and control of bifurcation problems part i: Bifurcation in finite dimensions. Int. J. Bifurcation and Chaos, 1(3):493-520, 1991.

[5] E. J. Doedel, H. B. Keller, and J.-P. Kernévez. Numerical analysis and control of bifurcation problems part ii: Bifurcation in finite dimensions. Int. J. Bifurcation and Chaos, 1(4):745-772, 1991.

[6] F. Dumortier and Chengzhi Li. Perturbations from an elliptic hamiltonian of degree four. i. saddle loop and two saddle cycle. J. Differential Equations, 176:114157, 2001.

[7] F. Dumortier and Chengzhi Li. Perturbations from an elliptic hamiltonian of degree four. ii. cuspidal loop. J. Differential Equations, 175:209243, 2001.

[8] F. Dumortier, Chengzhi Li, and Zifen Zhang. Unfolding of a quadratic integrable system with two centers and two unbounded heteroclinic loops. J. Differential Equations, 139:146193, 1997.

[9] F. Dumortier, D. Panazzolo, and R. Roussarie. More limit cycles than expected in liénard equations. Proc. Amer. Math. Soc., 135(6):1895-1904, 2007.

[10] J.L. Figueras, W. Tucker, and J. Villadelprat. Computer-assisted techniques for the verification of the chebyshev property of abelian integrals. J. Differential Equations, 254:3647-3663, 2013.

[11] J.-P. Françoise and C.C. Pugh. Keeping track of limit cycles. J. Differential Equations, 65(2):139-157, 1986.

[12] J.-P. Françoise and D. Xiao. Perturbation theory of a symmetric center within liénard equations. J. Differential Equations, 259(6):2408-2429, 2015.

[13] L. Gavrilov. Petrov modules and zeros of abelian integrals. Bull. Sci. Math., 122:571-584, 1998. 
[14] L. Gavrilov and I.D. Iliev. Perturbations of quadratic hamiltonian two-saddle cycles. Ann. Inst. H. Poincar Anal. Non Linaire, 32, 2015.

[15] M. Grau, F. Manosas, and J. Villadelprat. A chebyshev criterion for abelian integrals. Trans. Amer. Math. Soc., 363:109-129, 2011.

[16] J. Karamata. Sur quelques problmes poss par ramanujam. J. Indian Math. Soc., 24:343-365, 1960.

[17] Chengzhi Li and Zifen Zhang. A criterion for determining the monotonicity of the ratio of two abelian integrals. J. Differential Equations, 127:407-424, 1996.

[18] S. Lynch. Limit cycles of generalized linard equations. Appl. Math. Lett., 8(6):15-17, 1995.

[19] P. De Maesschalck and M. Desroches. Numerical continuation techniques for planar slow-fast systems. SIAM J. Appl. Dyn. Syst., 12(3):1159-1180, 2013.

[20] P. De Maesschalck and F. Dumortier. Bifurcations of multiple relaxation oscillations in polynomial linard equations. Proceedings of the American Mathematical Society, 139(6):2073-2085, 2011.

[21] P. De Maesschalck and M. Wechselberger. Neural excitability and singular bifurcations. J. of Math. Neuroscience, 5(1), 2015.

[22] Lin Ping Peng. Unfolding of a quadratic integrable system with a homoclinic loop. Acta Mathematica Sinica, 18:737-754, 2002.

[23] G. Petrov. Complex zeros of an elliptic integral. Funktsional. Anal. i Prilozhen, 21(3):87-88, 1987.

[24] S. Smale. Mathematical problems for the next century. Math. Intelligencer, 20(2):7-15, 1998. 